\title{
Sistema de cierre asistido por vacío en el tratamiento de la gangrena de Fournier: a propósito de un caso
}

Preciado-Estrella DA, Calvo-Vázquez I, Cortés-Raygoza PA, HernándezMéndez EA, y colaboradores.

\section{Resumen}

ANTECEDENTES: la gangrena de Fournier es un proceso inflamatorioinfeccioso, agudo y fulminante que produce necrosis de la piel y fascias del escroto, pene y periné, incluso puede provocar la muerte en $45 \%$ de los casos.

CASO CLÍNICO: paciente de 62 años de edad, sin alteraciones de importancia para el padecimiento actual, con antecedente de intervención quirúrgica por estenosis de uretra una semana previa al ingreso hospitalario. A la exploración física se encontró edema, eritema genital y perineal, que se extendía hacia ambas ingles e hipogastrio, sin afectación del ano. Las pruebas de laboratorio reportaron concentración de 32,000 leucocitos y bandemia con índice de severidad de 10 puntos de gangrena de Fournier. Se realizó debridación agresiva de los genitales y el periné, donde se encontró necrosis y exudado purulento que se infiltraba y extendía a las fascias de Camper, Scarpa y aponeurosis anterior hasta $10 \mathrm{~cm}$ por arriba de la sínfisis del pubis. Se continuó con debridamiento y a partir del tercer lavado se implementó el sistema de cierre asistido por vacío, hasta completar 5 lavados más en quirófano, con cambio del sistema. El paciente evolucionó adecuadamente, por lo que egresó a los 24 días postratamiento.

CONCLUSIÓN: el sistema de cierre asistido por vacío representa un método efectivo, útil y reproducible en pacientes con gangrena de Fournier. Garantiza buenos resultados funcionales y estéticos, además de disminuir las complicaciones y estancia hospitalarias.

PALABRAS CLAVE: gangrena de Fournier, sistema de cierre asistido por vacío.
División de Urología, Hospital General Dr. Manuel Gea González, Ciudad de México, México.

Recibido: diciembre 2016

Aceptado: julio 2017

Correspondencia

Dr. Diego Preciado Estrella

dr.diegopreciado@gmail.com

Este artículo debe citarse como

Preciado-Estrella DA, Calvo-Vázquez I, CortésRaygoza PA, y col. Sistema de cierre asistido por vacío en el tratamiento de la gangrena de Fournier: a propósito de un caso. Rev Mex Urol. 2017 julagos;77(4):312-317.

DOI: https://doi.org/10.24245/revmexurol.v77i4.1085 


\section{Vacuum-assisted closure system in the treatment of Fournier's gangrene: a case report}

\author{
Preciado-Estrella DA, Calvo-Vázquez I, Cortés-Raygoza PA, Hernández- \\ Méndez EA, et al.
}

\begin{abstract}
BACKGROUND: Fournier's gangrene is an acute and fulminating inflammatory-infectious process that produces necrosis of the skin and the fasciae of the scrotum, penis, and perineum. The disease can be fatal in up to $45 \%$ of the cases, and it requires multidisciplinary management.
\end{abstract}

CLINICAL CASE: A 62-year-old man, with no alterations relevant to his present condition, had undergone surgery due to urethral stricture one week prior to hospital admission. Physical examination revealed genital and perineal edema and erythema that extended to both inguinal regions and the hypogastrium, sparing the anus. Neutrophilia with 32,000 leukocytes and bandemia with a Fournier's Gangrene Severity Index of 10 points were reported in the laboratory work-up. Aggressive debridement of the genitals and perineum was performed, finding necrosis and purulent exudate that extended to and infiltrated the fascia of Camper, fascia of Scarpa, and the anterior aponeurosis up to $10 \mathrm{~cm}$ above the pubic symphysis. The debridement was continued, and from the third lavage, was managed through the vacuum-assisted closure system. Five more lavages were completed in the operating room and the devices were changed. The patient progressed adequately and was released after 24 days of treatment.

CONCLUSION: The use of vacuum-assisted closure devices is an effective, useful, and reproducible method that guarantees good functional and cosmetic results and reduces hospital stay and complications.

KEYWORDS: Fournier's gangrene; Vacuum-assisted closure device
Urology Division, Hospital General Dr. Manuel Gea González, Mexico, México.

Correspondence

Dr. Diego Preciado Estrella

dr.diegopreciado@gmail.com

\section{ANTECEDENTES}

La gangrena de Fournier es una alteración grave, descrita por primera vez por Baurienne en 1764, ${ }^{1}$ aunque se acredita el estudio extenso al venereólogo francés Jean Fournier, quien reportó las características clínicas que hoy conocemos. ${ }^{2}$
La gangrena de Fournier, también conocida como fascitis necrotizante del periné, es una enfermedad infecciosa que afecta la dermis y capas subcutáneas de la región anogenital, lo que resulta en mala calidad de vida del paciente. ${ }^{3}$ La epidemiología de la enfermedad varía según las poblaciones, pero la edad a la que 
se manifiesta ocurre entre los 40 y 70 años, con mayor predisposición en varones que en mujeres (proporción 10:1). En México se han identificado como principales factores de riesgo: nivel socioeconómico bajo, enfermedades crónicas (diabetes mellitus, hipertensión arterial, insuficiencia renal), alcoholismo, síndrome de inmunodeficiencia adquirida o enfermedades psiquiátricas. ${ }^{4}$ El alcoholismo es un factor importante y común en esta enfermedad, debido a su relación con inmunosupresión, mala higiene y estatus socioeconómico bajo. ${ }^{4}$

El tratamiento de primera línea incluye la rápida debridación quirúrgica y, en ocasiones, colostomía por derivación, o derivación urinaria mediante un catéter de cistostomía. ${ }^{5}$ En ocasiones, la evolución natural de la enfermedad puede ser muy agresiva, con desenlaces devastadores en la calidad de vida, por lo que es importante implementar estrategias para lograr mejores resultados funcionales.

Anteriormente, los tratamientos de la gangrena de Fournier incluían curaciones periódicas y debridación quirúrgica continua, para que cuando la herida estuviera limpia y en proceso de granulación, se consideraba el autotrasplante de tejidos y colgajos. Entre las opciones actuales destaca una técnica para aumentar la granulación, particularmente el dispositivo de cierre asistido por vacío $\left(\right.$ VAC $^{\circledR}$ Therapy por sus siglas en inglés), con recambio cada 48 a 72 horas.

El cierre de heridas asistido por presión negativa fue aprobado por la FDA (Food and Drug Administration $)^{6}$ en 1995, pues demostró excelentes resultados en el tratamiento de lesiones complejas. Los dispositivos comerciales disponibles incluyen los sistemas Chariker-Jeter y VAC (Vacuum assisted therapy), este último el más popular. Ambos sistemas consisten en una esponja de espuma, un sistema de recolección de fluidos, una cubierta adhesiva y una bomba de succión continua o intermitente. ${ }^{7}$

A continuación se informa un caso de gangrena de Fournier, tratado con un sistema de cierre asistido por vacío.

\section{CASO CLÍNICO}

Hombre de 62 años de edad, originario de una zona rural, sin enfermedades de importancia para el padecimiento actual, con antecedente de retención aguda de orina, quien acudió al servicio de Urgencias, donde se le colocó una sonda transuretral de forma traumática; 7 días después manifestó fascitis necrotizante (gangrena de Fournier). A la exploración física se encontró taquicardia (98 latidos por minuto), fiebre $\left(39.6^{\circ} \mathrm{C}\right)$ y polipnea (24 respiraciones por minuto); se observó edema y eritema genital, que se extendía a la región perineal, hipogastrio, área inguinal bilateral y ambos flancos, sin afectación del esfínter anal y los glúteos (Figura 1).

Los estudios de laboratorio reportaron: citometría hemática con 32,000 leucocitos y bandemia (93\% de polimorfonucleares); química sanguínea con elevación de azoados (creatinina de $2.2 \mathrm{mg} / \mathrm{dL}$, urea de $96 \mathrm{mg} / \mathrm{dL}$ ) y trastorno hidroelectrolítico a expensas de hipernatremia, con índice de severidad estimado de 10 puntos de gangrena de Fournier. ${ }^{8}$

Se realizó debridación quirúrgica agresiva de genitales y periné, pues se encontró necrosis con exudado purulento, que infiltraba hacia las fascias de Camper y Scarpa, y aponeurosis anterior hasta $10 \mathrm{~cm}$ por arriba de la sínfisis del pubis, delimitándose hasta encontrar tejido sano (Figura 2). Se inició tratamiento con sistema de cierre asistido por vacío a partir del tercer lavado de la herida, con cambio cada 48 a 72 horas mediante presiones intermitentes de $75 \mathrm{mmHg}$. (Figura 3). El paciente egresó 24 días después de estancia 


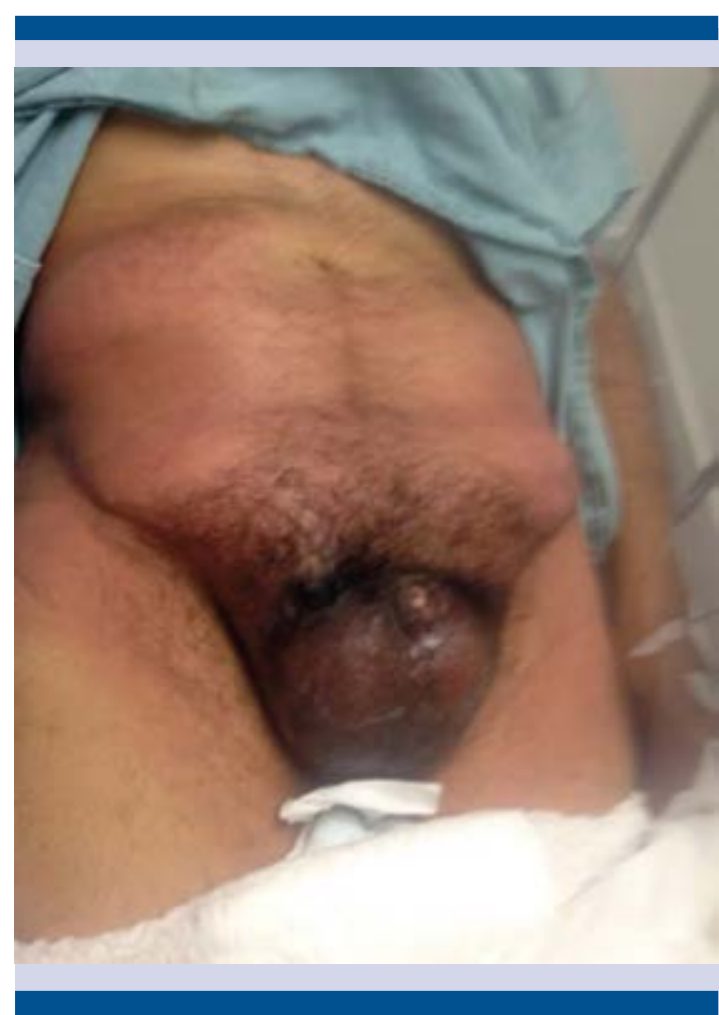

Figura 1. Aspecto clínico del paciente al ingreso hospitalario.

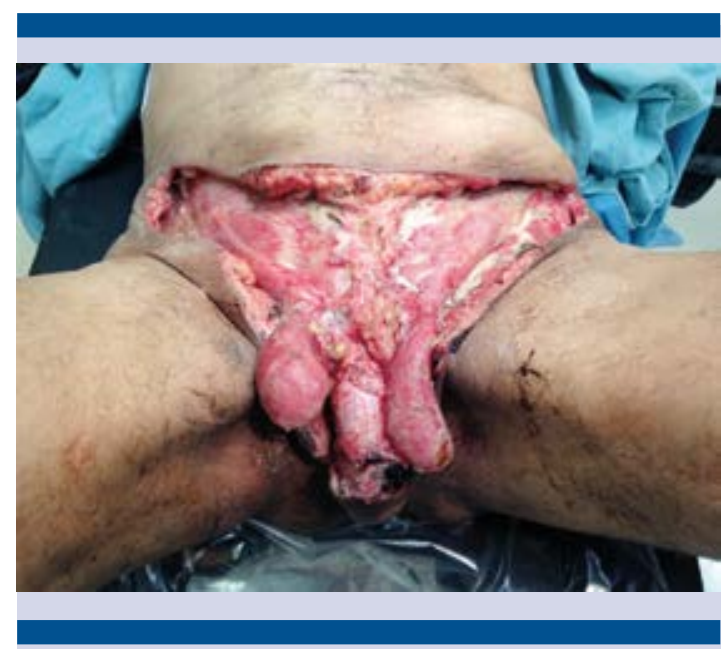

Figura 2. Debridación después del primer tiempo quirúrgico.

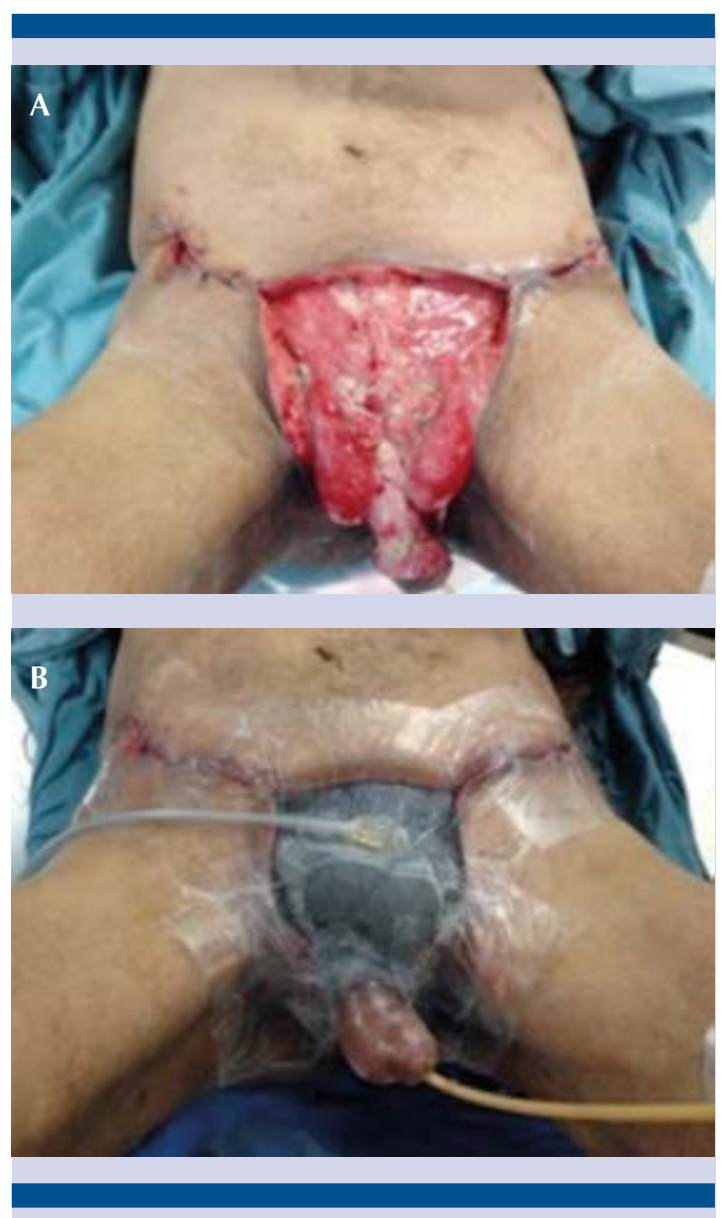

Figura 3. Colgajos de avance y granulación de la herida (A); cierre de la herida mediante el sistema de vacío (B).

hospitalaria e inició tratamiento ambulatorio con el mismo sistema, cada 72 horas, hasta alcanzar el cierre de $75 \%$ de la herida, para después colocar colgajos cutáneos de avance (Figura 4).

\section{DISCUSIÓN}

La gangrena de Fournier es una alteración compleja que requiere soporte hemodinámico, prescripción de antibióticos, intervención quirúrgica agresiva y tratamiento de la herida por médicos experimentados. Dada su complejidad, 


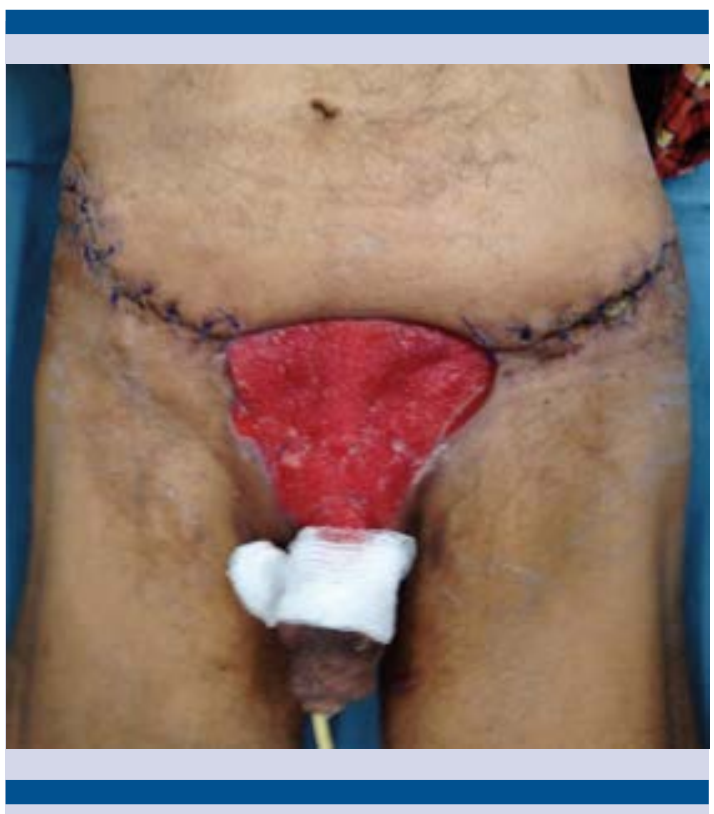

Figura 4. Aspecto del paciente al egreso hospitalario.

además de las malas condiciones de los pacientes, la pérdida de tejido y las complicaciones, el tiempo de recuperación suele ser prolongado.

La infección local, especialmente cuando se asocia con afección inmunitaria, puede propagarse desde la fascia de Buck, que cubre las estructuras más profundas del pene y la región periuretral, hacia la fascia de Colles y dartos, incluso extenderse a planos más profundos. ${ }^{9}$

Los sistemas de cierre asistidos por vacío ofrecen múltiples beneficios para acelerar el proceso de granulación y cierre de la herida en cualquier parte del cuerpo; ${ }^{10}$ por ejemplo:

- Aceleran la formación de tejido de granulación, estimulan la perfusión sanguínea con neovascularización y favorecen la cicatrización.

- Mantienen la herida en un microambiente dinámico e impiden la colonización bacteriana, pues los microorganismos aerobios y anaerobios sinergizan la coagulación microvascular e hipoxia tisular. ${ }^{11}$

- Estimulan la laxitud del tejido al acercar los bordes hasta conseguir confrontarlos, mediante la estimulación de colonias de fibroblastos.

Si bien algunas series demuestran que los sistemas de cierre asistidos por vacío reducen el tiempo de recuperación, otras señalan que éste es mayor; lo cierto es que mejoran los resultados funcionales y el costo-beneficio a largo plazo, favoreciendo la incorporación temprana del paciente a su vida laboral. Czymek y su grupo compararon prospectivamente datos de $35 \mathrm{pa}$ cientes tratados con sistema de cierre asistido por vacío versus tratamiento convencional (lavado y apósitos antisépticos diarios) y encontraron menor mortalidad en el primer grupo, pero mayor estancia hospitalaria. ${ }^{12}$

Por su parte, Ozturk y sus coautores publicaron una serie de 10 pacientes tratados con sistema de cierre asistido por vacío y los compararon con sujetos que recibieron terapia convencional. Entre sus resultados encontraron costos similares con ambos protocolos, pero beneficios superiores con el sistema de cierre asistido por vacío en cuanto a disminución del dolor, mayor capacidad de movimiento, ausencia de dieta y satisfacción del paciente y los médicos tratantes. ${ }^{13}$

Los sistemas de cierre asistidos por vacío representan una alternativa efectiva y segura en el tratamiento de la gangrena de Fournier. Su aplicación es sencilla y las instituciones de salud con experiencia brindan excelentes resultados funcionales y estéticos, disminuyen el tiempo de estancia hospitalaria y las complicaciones, además de implementar un tratamiento ambulatorio y reducir los costos médicos a largo plazo. 


\section{Financiamiento}

No se requirió ningún tipo de financiamiento para realizar este trabajo.

\section{Declaración de conflicto de intereses}

No existe conflicto de interés.

\section{REFERENCIAS}

1. Torremadé-Barreda $T$, Millán-Scheiding $M$, Suárez-Fernández C, Cuadrado-Campaña JM, Rodríguez-Aguilera JJ, Franco-Miranda E. Gangrena de Fournier: Estudio retrospectivo de 41 casos. Cir Esp 2010;87:218-223.

2. Forunier JA. Jean-Alfred 1832-1914. Gangéne foudroyante de la verge. Sem Med 1883. Dis Colon Rectum 1988;31(12):984-8.

3. Ozkan OF, Altynly E, Koksal N, Senger S, Celik A. Combining Flexi-Seal and negative pressure wound therapy for wound management in Fournier's gangrene. Int Wound J 2013;12(3):364-5.

4. Sedano-Basilio JE, Cornejo-Dávila L, Trujillo-Ortiz M, Cantellano-Orozco $\mathrm{G}$, et al. Experiencia y revisión de la literatura en el manejo de gangrena de Fournier en una institución, 2008-2015. Rev Mex Urol 2016;76(1):29-35.

5. Bordes J. A non-surgical device for faecal diversion in the management of perineal burns. Burns 2008;34:840844.

6. Vacuum Assisted Closure Wound Therapy Cleared for Partial Thickness Burns". Reuters Health Medical News. January 27, 2003.

7. Venturi ML. Mechanisms and clinical applications of the vacuum-assisted closure (VAC) Device: a review. Am J Clin Dermatol. 2005;6(3):185.

8. Laor E, Palmer LS, Tolia BM, Reid RE, Winter Hi. outcome prediction in patients with fourniers gangrene. J Urol 1995;154:89-92.

9. Yaghan RJ, Al-Jaberi TM, Bani-Hani I, et al. Fournier's gangrene. Dis Colon Rectum 2000;43:1300-8.

10. Fogg E. Best treatment of nonhealing and problematic wounds. JAAAPA 2009;22(8):46, 48.

11. Giuliano A, Lewis F Jr, Hadley K, et al. Bacteriology of necrotizing fasciitis. Am J Surg 1977; 134:52-7.

12. Czymek R, Schmidt A, Eckmann C, Bouchard R, et al Fournier's gangrene: vacuum-assisted closure versus conventional dressings. Am J Sur 2009;197(2):168-76.

13. Ozturk, Ozguc $\mathrm{H}$, Yilmazlar T. The use of vacuum assisted closure therapy in the management of Fournier's gangrene. Am J Surg 2009;197(5):660-5.

\section{AVISO PARA LOS AUTORES}

Revista Mexicana de Urología tiene una nueva plataforma de gestión para envío de artículos: https://www.revisionporpares.com/index.php/RMUrol ahí podrá inscribirse a la base de datos administrada por el sistema Open Journal System (OJS) que ofrece las siguientes ventajas para los autores:

- Subir sus artículos directamente al sistema.

- Conocer, en cualquier momento, el estado de los artículos enviados, es decir, si ya fueron asignados a un revisor, aceptados con o sin cambios, o rechazados.

- Participar en el proceso editorial corrigiendo y modificando sus artículos hasta su aceptación final. 